\title{
Does one night of partial sleep deprivation affect the evening performance during intermittent exercise in Taekwondo players?
}

\author{
Mohamed Arbi Mejri' ${ }^{1,2 *}$, Narimen Yousfi ${ }^{1,3}$, Thouraya Mhenni ${ }^{1,2}$, Amel Tayech ${ }^{1,3}$, Omar Hammouda ${ }^{4}$, Tarak Driss ${ }^{4}$, Anis Chaouachi', \\ Nizar Souissi ${ }^{1,5}$
}

'Research Laboratory "Sport Performance Optimization", National Center of Medicine and Sciences in Sport (CNMSS), Tunis, Tunisia

${ }^{2}$ Faculty of Science, Carthage University, Bizerte, Tunisia

${ }^{3}$ High Institute of Sport and Physical Education, Ksar-Saïd, Manouba University, Tunis, Tunisia

${ }^{4}$ Université Paris Ouest Nanterre La Défense, CeRSM (EA 2931), UFR STAPS, Nanterre, France

${ }^{5}$ National Observatory of Sport, Tunis, Tunisia

Athletes and coaches believe that adequate sleep is essential for peak performance. There is ample scientific evidence which support the conclusion that sleep loss seems to stress many physiological functions in humans. The aim of this study was to determine the effect of one night's sleep deprivation on intermittent exercise performance in the evening of the following day. Ten male Taekwondo players performed the $Y_{0}-Y_{0}$ intermittent recovery test (YYIRT) in three sleep conditions (reference sleep night [RN], partial sleep deprivation at the beginning of night [PSDBN], partial sleep deprivation at the end of night [PSDEN]) in a counterbalanced order, allowing a recovery period $\geq 36 \mathrm{hr}$ in between them. Heart rate peak $\left(H \mathrm{HR}_{\text {peak }}\right)$, plasma lactate concentrations (Lac) and rating of perceived exertion (RPE) were measured during the test. $A$ significant effect of sleep restriction was observed on the total distance covered in YYIRT $(P<0.0005)$ and $\operatorname{Lac}(P<0.01)$ in comparison with the RN. In addition, performance more decreased after PSDEN $(P<0.0005)$ than PSDBN $(P<0.05)$. Also, Lac decreased significantly only after PSDEN $(P<0.05)$ compared with RN. However, there were no significant changes in $\mathrm{HR}_{\text {peak }}$ and RPE after the two types of partial sleep deprivation compared to RN. The present study indicates that short-term sleep restriction affect the intermittent performance, as well as the Lac levels of the Taekwondo players in the evening of the following day, without alteration of $\mathrm{HR}_{\text {peak }}$ and $\mathrm{RPE}$.

Keywords: Sleep loss, Evening performance, Intermittent exercise, Endurance intensive effort, Yo-Yo test, Taekwondo

\section{INTRODUCTION}

One of the most fundamental rhythms is the sleep-wakefulness cycle (Reilly and Edwards, 2007). Indeed, recent studies have demonstrated that sleep has an integral role in metabolic homeostasis (Xie et al., 2013). For athletes, it has been evidenced that adequate sleep is generally associated with better physical performances (Davenne, 2009; Mejri et al., 2014b; Souissi et al., 2008; Souissi et al., 2013) and adequate recovery from competitions despite they are faced to sleep deprivation (SD) situations induced before or during a competition (i.e., state of stress and anxiety preceding the competition, activities for a period which exceeds $24 \mathrm{hr}$ such as ultratriathlon, time difference syndrome "Jet-lag") (Davenne, 2009; Mejri et al., 2014b; Reilly and Edwards, 2007; Souissi et al., 2008). Thus, since sleep disruption prior to important events is commonly found in elite athletes (Juliff et al., 2015). In this regards, recent studies revealed that sleep disturbance is generally accompanied with an "underperformance" during the competition (Reilly and Edwards, 2007; Souissi et al., 2008). Recently numerous studies supported the conclusion that partial sleep deprivation (PSD) impaired anaerobic performance in the afternoon, but not in the morning (Souissi et al., 2008).
${ }^{*}$ Corresponding author: Mohamed Arbi Mejri (D http://orcid.org/0000-0001-7966-6689 Research Laboratory “Sport Performance Optimization”, National Center of Medicine and Sciences in Sport (CNMSS), Ave Med Ali Akid, 1004 El Menzah, Tunis, Tunisia Tel: +216-55731648, Fax: +216-70709789, E-mail: mejriarbi@gmail.com

Received: November 21, 2015 / Accepted: February 2, 2016
This is an Open Access article distributed under the terms of the Creative Commons Attribution Non-Commercial License (http://creativecommons.org/licenses/by-nc/4.0/) which permits unrestricted non-commercial use, distribution, and reproduction in any medium, provided the original work is properly cited. 
However, in aerobic performance previous investigations reported a decrease (Azboy and Kaygisiz, 2009; Chen, 1991; Oliver et al., 2009; Plyley et al., 1987; Ricardo et al., 2009), or no modification (Azboy and Kaygisiz, 2009; Chen, 1991; Goodman et al., 1989; Oliver et al., 2009; Racinais et al., 2004) after a total sleep deprivation (TSD) which is almost never occurred in athletes despite that just few studies (Mougin et al., 1991; Mougin et al., 2001; Omiya et al., 2009) have investigated the effects of PSD on aerobic performance, and reported contradictory findings. The discrepancy observed in earlier studies could be explain by the differences in populations, duration of SD, and the performance assessment protocols as mention Racinais et al. (2004).

On the other hand, recently Mejri et al. (2014c) studied in ten male Taekwondo players the effects of two PSD conditions on morning performance of an endurance intensive effort as mentioned by Chamari and Padulo (2015). This last couldn't find any significant effect of SD on the Yo-Yo intermittent recovery test (YYIRT) morning performance. Moreover, according to Bouhlel et al. (2006), Taekwondo competition has been classified as an intermittent maximal exercise which stresses simultaneously aerobic and anaerobic metabolisms. On the other hand, the physiological measurements performed during the YYIRT indicated that the aerobic energy turnover reached maximal values and the anaerobic energy system was highly taxed toward the end of the test (Bangsbo et al., 2008; Krustrup et al., 2003), which made it a suitable test for Taekwondo players. In light of the above considerations and since Taekwondo training sessions where generally organized in the evening, one speculates that research interested with performance among Taekwondo players have to focus on the effect of PSD in the evening.

To address the limited knowledgebase pertaining of the effect of PSD on endurance intensive performance during field intermittent exercises, we aimed to examine the effects of the two types of PSD (delayed bedtime and early awakening) on Taekwondo players' evening performance after an intermittent exercise.

\section{MATERIALS AND METHODS}

\section{Participants}

Ten healthy male Taekwondo players (age, $17.6 \pm 0.52 \mathrm{yr}$; height, $175.8 \pm 6.21 \mathrm{~cm}$; body mass, $61.17 \pm 5.80 \mathrm{~kg}$; \% body Fat, $9.88 \% \pm 1.77 \%$; body mass index, $19.22 \pm 2.82 \mathrm{~kg} / \mathrm{m}^{2}$ ) enrolled in the study as volunteers. They were competitors at national standard with black belt and $6.60 \pm 1.26 \mathrm{yr}$ (mean \pm standard deviation [SD]) of experience in TKD practices. They usually trained at least three days/week for an average of $2 \mathrm{hr}$ daily evening training. Prior to starting the study, all subjects were given a through explanation of the experimental procedures and the possible risks and discomforts associated with the study. Each participant signed a written informed consent form prior to participation. Participants were screened by medical history and physical examinations including any history of severe physical or mental disorder. They were also selected based on the exact inclusion/exclusion criteria:

- They were trained subjects;

- They reported no use of alcohol or tobacco and none of them was taking antioxidant compounds, including vitamins and medications (e.g., anti-inflammatory agents);

- They were also selected based on their chronotype using the questionnaire of Horne and Ostberg (1976) (only subjects of "intermediate" $[\mathrm{n}=7]$ or "moderate morning" $[\mathrm{n}=3]$ types were included to this study);

- They do not have history of sleep disorders and they had also regular sleeping schedules based on the Bastuji and Jouvet (1985) calendar, completed over 1-month period prior to the investigation (mean $\pm \mathrm{SD}$ ) estimated sleep duration was $8.2 \pm 0.9 \mathrm{hr}$ (hour \pm fraction).

The study protocol complied with the Helsinki declaration for human experimentation and was approved by the Clinical Research Ethics Committee of the National Centre of Medicine and Science of Sports of Tunis (CNMSS) before the starting of the assessments.

\section{Procedures}

During the week before the experiment, all participants were fully familiarized with the procedure and tests involved to minimize learning effects during the experiment. All experimental conditions for sleep were carried out identically in a sports science laboratory. Participants were regularly sleeping between 10:30 p.m. and 6 a.m. in the laboratory, in an individual temperature-controlled bedroom $\left(22^{\circ} \mathrm{C}-24^{\circ} \mathrm{C}\right)$ where the ambient conditions were maintained as constant as possible. To familiarize participants with the laboratory conditions, the first experimental day and night were used as adaptation only. Then, the subjects completed three experimental sleep conditions, in a random, counterbalanced order, allowing recovery period $\geq 36 \mathrm{hr}$ in between them. During each session, and to control and standardize their diet and activities, each participant arrived at the laboratory at 7 a.m. and stayed overnight. The first condition was a reference night $(\mathrm{RN})$ during which the participants were synchronized with 
a nocturnal sleep of about $7 \mathrm{hr}$, in the period between 10:30 p.m. and 6 a.m. The second condition was a of PSD in the beginning of the night (PSDBN) during which subjects were deprived of about $4 \mathrm{hr}$ of sleep at the beginning of the night, sleeping for about 3 hr only (between 3 a.m. and 6 a.m.), sleep being prohibited before and after this period of time. The third condition was a PSD in the end of the night (PSDEN) during which they were also deprived of about $3 \mathrm{hr}$ of sleep, but at the end of the night, sleeping only about $4 \mathrm{hr}$ (between 10:30 p.m. and 3 a..m). They were not allowed to sleep before or after this period of time.

Participants were not allowed to sleep after woken up or before bed time. To keep them awake, when required during both SD protocols, laboratory illumination was maintained at 150-200 lux. They were allowed to watch television, read books, work on a computer, listen to music, or converse with the staff or visitors during these times, but were not allowed to ingest food, caffeine or stimulants, and they were observed continuously by a technician. During the three sleep conditions and after their waking, subjects were not allowed to spend any other time in bed or use opportunities for naps.

After each sleep condition, all subjects performed one test session in the evening ( 5 p.m.) of the following day. Instructions about sleep and diet were given to the subjects prior to the experiment. During each condition, they were prescribed standard isocaloric diet ( $40 \%$ of energy from fat, $16 \%$ protein, and $44 \%$ carbohydrates). Meals were served at fixed times: 7 a.m. (breakfast), 12 p.m. (lunch), and 8 p.m. (dinner). After dinner, only fresh water was allowed ad libitum prior to sleep. Likewise, on the following day, after lunch, just fresh water was allowed ad libitum prior to the 5 p.m. test. Throughout the experimental period, the subjects were not allowed to eat or drink any caffeinated or supplements and other psychoactive substances. They were requested to maintain their habitual physical activity throughout the experimental period and to avoid strenuous activity during the $24 \mathrm{hr}$ before the test sessions. Compliance with directions relating to pretest sleep, activity, and diet was checked by daily activity and dietary diaries. Subjects' body mass, height, and percentage of body fat (skinfolds) were recorded just before the first test session using BC-418 Segmental Body Composition Analyzer (Tanita, Tokyo, Japan)

During each test session, they were asked to perform the YYIRT level 1 in the indoor sports hall. They were instructed and verbally encouraged to give maximal effort during this test. A standardized warm-up consisting of jogging, dynamic stretching, and increasing intensity sprints was performed before testing.
Heart rate peak $\left(\mathrm{HR}_{\text {peak }}\right)$ was recorded during the YYIRT using a Polar heart rate monitor (T61-coded, Polar Electro Oy, Kempele, Finland). Blood samples were collected from a forearm vein 3 min after the YYIRT. A blood collection tube with sodium fluoride was used for the measurement of plasma lactate concentrations (Lac).

\section{Measurements and protocols \\ The YYIRT level 1}

As previously described (Mejri et al., 2014c), the YYIRT was performed according the procedures suggested by Krustrup et al. (2003). The test consisted of 20-m shuttle runs performed at increasing speeds with $10 \mathrm{sec}$ of active recovery in a distance of $5 \mathrm{~m}$ between runs until exhaustion. Audio cues of the YYIRT were recorded on a CD (www.teknosport.com, Ancona, Italy) and broadcasted using a portable CD player (Az1030 CD player, Philips, Eindhoven, Holland). The end of the test was considered when the participant twice failed to reach the front line in time (objective evaluation) or he felt unable to complete another shuttle at the dictated speed (subjective evaluation). The total distance (TD ${ }_{\text {YYIRT }}$ ) covered during the YYIRT was considered as the test score. Although, this test is used to evaluate an athletes' ability to repeatedly perform intense exercise and his potential to recover from intense exercise. Before the test, all participants carried out a warm up period consisting of the first four running bouts in the test. The total duration of the test was 6-20 min. All participants were already familiar with the testing procedures as it was part of their usual fitness assessment program.

\section{Rating of perceived exertion scale}

Rating of perceived exertion (RPE) was determined at the end of the YYIRT. The RPE scale allows participants to give a subjective exertion rating for the physical task. It consists of a 15-point scale ranging from 6 (very, very light) to 20 (very, very hard). The RPE scale is a reliable indicator of physical discomfort, has sound psychometric properties, and is strongly correlated with several other physiological measures of exertion (Borg, 1982).

\section{Statistical analyses}

All statistical tests were processed using the IBM SPSS Statistics ver. 19.0 (IBM Co., Armonk, NY, USA). All values within the text and figures are reported as the mean and standard deviation (mean $\pm \mathrm{SD}$ ). The Shapiro-Wilk W-test revealed that data were normally distributed. Once the assumption of normality was confirmed, parametric tests were performed. Data obtained 
during maximal exercise following the two conditions of sleep deprivation (PSDBN, PSDEN) were compared to those obtained after the RN. Data were analyzed using a one-way analysis of variance (ANOVA) with repeated measures (three [sleep condition]). When the ANOVA indicated significant sleep condition effect, post hoc multiple comparisons using the least significant difference (LSD) Fischer test was conducted. Effect sizes (ES) were calculated as partial eta-squared $\eta_{\mathrm{p}}{ }^{2}$ to assess the practical significance of our findings. ES was interpreted as follows: $\sim 0.2$, small; $\sim 0.5$, moderate; and $\sim 0.8$, large. The level of significance was set at $P<0.05$. When the SPSS output demonstrated significance levels of $P=0.000$, these were corrected to $P<0.0005$.

\section{RESULTS}

Mean values of TD $\mathrm{TD}_{\text {YIRT }}(\mathrm{A}), \mathrm{HR}_{\text {peak }}(\mathrm{B}), \mathrm{Lac}(\mathrm{C})$, and RPE (D) recorded after the three experimental conditions, are presented in Fig. 1. There was a significant effect of sleep condition for TDYYIRT $\left(F_{(2.18)}=23.776, P<0.0005, \eta_{\mathrm{p}}{ }^{2}=0.73\right)$ and $\operatorname{Lac}\left(F_{(2.18)}=8.477\right.$,
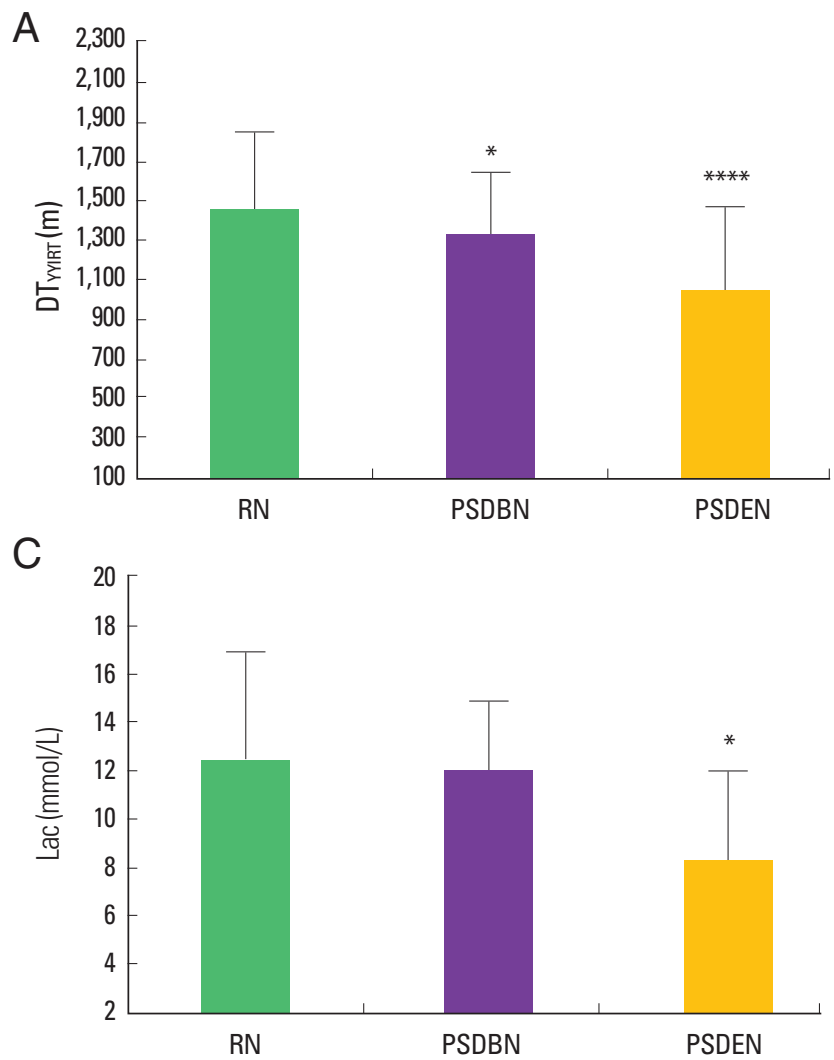

$\left.P=0.006, \eta_{\mathrm{p}}{ }^{2}=0.50\right)$. The post hoc analysis revealed that these TDYYIRT decreased significantly after PSDBN $(P<0.05)$ and PSDEN $(P<0.0005)$ compared with RN (Fig. 1A). Indeed, the decline of performance was more pronounced after PSDEN than PSDBN $(P<0.01)$. Likewise, Lac concentrations decreased significantly only after PSDEN $(P<0.05)$ compared with RN (Fig. 1C). However, there was no significant changes in $\operatorname{HR}_{\text {peak }}\left(F_{(2.18)}=0.887\right.$, $\left.P=0.391, \eta_{\mathrm{p}}{ }^{2}=0.09\right)$ (Fig. 1B) and RPE $\left(F_{(2.18)}=0.039, P=0.927\right.$, $\left.\eta_{\mathrm{p}}{ }^{2}=0.004\right)$ (Fig. 1D) after the two types of PSD compared to RN.

\section{DISCUSSION}

The main purpose of this study was to investigate the effects of two different types of PSD on Taekwondo players' performance during an intermittent exercise in the evening of the following day. To the best of our knowledge, no study has evaluated the effects of PSD on performance measures during an intermittent field test in the evening of the following day. In the current study, the effect of PSD on the evening intermittent performance was

$\mathrm{B}$

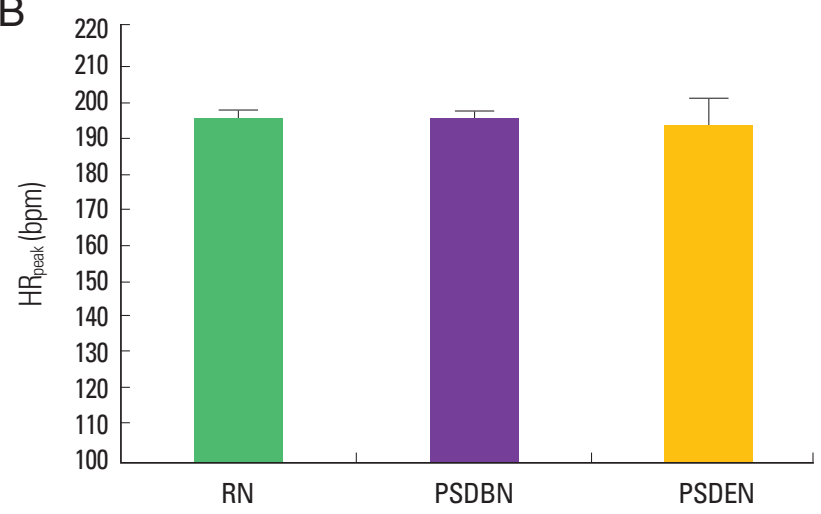

$\mathrm{D}$

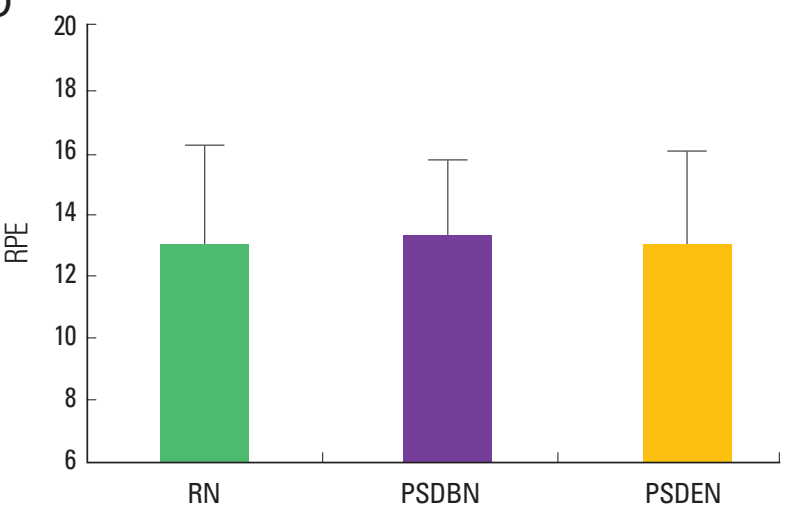

Fig. 1. Yo-Yo intermittent recovery test (YYIRT) performances (total distance covered during the YYIRT [TD YIRT] $_{\text {[ }}$ [A], heart rate peak [HR peak $_{\text {] }}[\mathrm{B}]$, plasma lactate concentrations [Lac] [C], and rating of perceived exertion scale [RPE] [D]) recorded during the three experimental conditions (reference night [RN], partial sleep deprivation in the beginning of the night [PSDBN], and partial sleep deprivation in the end of the night [PSDEN]). ${ }^{* * * * *}$ Significant difference compared to $\mathrm{RN}(P<0.05, P<0.0005$, respectively). 
evaluated with the same study population using an endurance intensive exercise field test (i.e., YYIRT) specifically designed to evaluate the ability to perform high-intensity intermittent exercise (Bangsbo et al., 2008; Krustrup et al., 2003). As a result, we come up with a significant decline in evening performance (i.e., TD $D_{\text {YYIRT }}$ ) following the two types of PSD. Moreover, a significant decrease in the values of Lac was observed only following PSDEN, compared with RN. However, the present results showed that one night of PSD when timed at the beginning or at the end of the night exerted no effect on $\mathrm{HR}_{\text {peak }}$ and $\mathrm{RPE}$ responses during the YYIRT.

In previous studies, the effects of $\mathrm{SD}$ on endurance intensive performances was examined after submaximal (Martin and Chen, 1984; Mougin et al., 1991; Mougin et al., 2001; Oliver et al., 2009; Ricardo et al., 2009), maximal (Azboy and Kaygisiz, 2009; Chen, 1991; Goodman et al., 1989; Martin and Chen, 1984; Mougin et al., 1991; Mougin et al., 2001; Mejri et al., 2014c; Oliver et al., 2009; Omiya et al., 2009; Plyley et al., 1987; Racinais et al., 2004; Ricardo et al., 2009) exercise. However, conflicting findings were observed and that could be mainly attributed to differences in exercise protocols, participants' fitness, experimental environment, and certainly, durations of the SD.

Regarding the effect of SD on evening endurance intensive performances (i.e., TD $D_{\text {YYRT }}$ ), the results of this work are in agreement with findings of previous studies, showing a significant effect of TSD (Azboy and Kaygisiz, 2009; Chen, 1991; Martin and Chen, 1984; Oliver et al., 2009; Plyley et al., 1987; Ricardo et al., 2009) and/or PSD (Mougin et al., 1991; Mougin et al., 2001) on endurance intensive performances. In this context, and similar to the SD protocol of the present study, Mougin et al. (1991) examined in seven male cyclists the effect of $3 \mathrm{hr}$ of PSD in the middle of night on afternoon (i.e., 2 p.m.) performance during an incremental exercise on a cycle ergometer, and showed a significant decrease in maximal oxygen uptake $\left(\mathrm{VO}_{2} \mathrm{max}\right)$. Recently, Mougin et al. (2001) revealed a decrease in afternoon maximal work rate among highly trained male athletes during an incremental exercise on a cycle ergometer, after two types of PSD (i.e., PSDBN and PSDEN) compared to RN. This might be explained by the higher muscle fatigue in the evening compared with the morning (Chtourou et al., 2013). Hence, this fatigue could be more important in the evening due to the rise of the resting biological markers of muscle injury and oxidative stress status in this time-of-day, and a more efficient antioxidant status in the morning than the evening (Hammouda et al., 2012). On the other hand, this current study supports the conclusion that PSDEN was more disturbing than the other types of PSD (Jarraya et al., 2013; Mougin et al., 2001; Mejri et al., 2014a, 2014c; Souissi et al., 2008; Souissi et al., 2013). Indeed, the adverse effects of this type of PSD (i.e., PSDEN) may be due to the specific characteristics of this private phase of sleep, and the current finding confirms the authors' recent results showing that PSDEN, unlike PSDBN, alters the resting level of monocytes (Mejri et al., 2014a) and increases the biomarkers of cardiac damage responses, at rest (i.e., creatine phosphokinase and myoglobin) (Mejri et al., 2015) in comparison with a RN. Otherwise, Jarraya et al. (2013) clarified that the adverse effect of the PSDEN could be explained by the absence of paradoxical sleep, which increases at the end of night to involved in the synchronization of the circadian system. On the other hand, the present findings are in disagreement with previous studies which found no modification in endurance intensive performances after SD (Azboy and Kaygisiz, 2009; Chen, 1991; Goodman et al., 1989; Mejri et al., 2014c; Mougin et al., 1991; Oliver et al., 2009; Omiya et al., 2009; Racinais et al., 2004). These contradictory results could be explain by the differences in fitness level of participants, durations and intensities of the exercise (i.e., continuous exercise, submaximal, or maximal exercise until exhaustion, intermittent exercise), time-of-day of measurements (i.e., morning, afternoon, evening), as well as types and durations of SD (i.e., TSD, PSD in the beginning, the middle, or the end of night).

Concerning the Lac concentrations and in accordance with previous studies (Mougin et al., 1991; Mougin et al., 2001), the present data showed that PSDEN affected significantly the Lac levels in response to endurance intensive effort (i.e., YYIRT) in trained subjects. However, these studies cited above found a significant increase in Lac levels following SD. It seems that the decline of the athletes' performance during YYIRT following the PSDEN, might be the cause of this Lac alteration in response to the exercise. On the other hand, the present study results are in disagreement with the previous reports which demonstrated that SD did not change on exercise Lac (Goodman et al., 1989; Martin and Chen, 1984; Mougin et al., 2001; Plyley et al., 1987). In view of the above considerations, several methodological issues between the studies cited above and the current study may contribute to the inconsistent findings for Lac responses. On the other side, we speculate that these alterations (i.e., TD TYIRT, Lac) observed following the night of PSD may be the result of inflammatory reactions induced by lack of sleep. In this regard, it has been well established that one night of PSD triggers inflammatory reactions (Irwin et al., 1996; Mejri et al., 2014a; Mejri et al., 2015), and can even deplete the immune system during the evening, es- 
pecially among young athletes (Mejri et al., 2014a).

The present findings showed that no change was spotted in $\mathrm{HR}_{\text {peak }}$ and RPE in responses to YYIRT following the two types of PSD. These results are well in agreement with other published investigations which revealed that did not change exercise HR (Azboy and Kaygisiz, 2009; Chen, 1991; Goodman et al., 1989; Martin and Chen, 1984; Martin and Gaddis, 1981; Meney et al., 1998; Mejri et al., 2014c; Oliver et al., 2009; Omiya et al., 2009; Plyley et al., 1987; Ricardo et al., 2009) and RPE (Meney et al., 1998; Mejri et al., 2014c; Oliver et al., 2009). This last result affirmed the hypothesis that RPE is normally closely related to HR (Borg, 1971). Otherwise, the present findings are in disagreement with the previous reports which showed that $\mathrm{SD}$ (i.e., total or partial) affected (i.e., decrease or increase) exercise HR (Chen, 1991; Mougin et al., 1991; Oliver et al., 2009; Omiya et al., 2009; Plyley et al., 1987) and increased RPE (Martin and Gaddis, 1981; Plyley et al., 1987). This controversy between results seems to be logical since in all of these studies it was noted that the population was not all athletes (sedentary or physically active), while the population of the present study composed from athletes. In addition, SD in those studies was total ( $>24 \mathrm{hr}$ ), while in the present study, we investigated one night PSD (Azboy and Kaygisiz, 2009; Goodman et al., 1989; Oliver et al., 2009; Plyley et al., 1987; Ricardo et al., 2009). Furthermore, there is a big difference between submaximal or maximal continuous tests performed in a laboratory on treadmill or cycle-ergometer (Azboy and Kaygisiz, 2009; Goodman et al., 1989; Oliver et al., 2009; Plyley et al., 1987; Ricardo et al., 2009) and the intermittent field test (YYIRT) performed in the current study. In this field test, a recovery period is incorporated after each pair of 20-m shuttle runs. In fact, this test evaluates the ability to repeat high intensity aerobic work and the anaerobic energy system was highly taxed toward the end of the test (Bangsbo et al., 2008; Krustrup et al., 2003).

In summary, results from this study suggest that one night of PSD, when timed at the beginning or at the end of the night decreased the Taekwondo players' intermittent performance (i.e., YYIRT) in the evening of the following day, whereas no alterations in $\mathrm{HR}_{\text {peak }}$ and RPE were observed following either type of PSD. In addition, it seems reasonable to conclude that the significant decrease of Lac concentrations observed following PSDEN, reflects also that PSDEN is more disturbing than PSDBN. The results of the present study must warn athletes practicing intermittent sports (racket and ball games, Taekwondo, Karate, boxing, Kung Fu, and Kick-boxing), that one night of PSDBN or especially PSDEN before a competition constitute a disturbance agent of high risk on subsequent performance.

\section{CONFLICT OF INTEREST}

No potential conflict of interest relevant to this article was reported

\section{ACKNOWLEDGMENTS}

The authors wish to express their sincere gratitude to all the participants for their maximal effort and cooperation. This study was supported by The Research Laboratory "Sports Performance Optimization" National Center of Medicine and Science in Sports (CNMSS).

\section{REFERENCES}

Azboy O, Kaygisiz Z. Effects of sleep deprivation on cardiorespiratory functions of the runners and volleyball players during rest and exercise. Acta Physiol Hung 2009;96:29-36.

Bangsbo J, Iaia FM, Krustrup P. The Yo-Yo intermittent recovery test: a useful tool for evaluation of physical performance in intermittent sports. Sports Med 2008;38:37-51.

Bastuji $\mathrm{H}$, Jouvet $\mathrm{M}$. Intérêt de l'agenda de sommeil pour l'étude des troubles de la vigilance. Electroencephalogr Clin Neurophysiol 1985;60:299305.

Borg G. The perception of physical performance. In: Shephard RJ, editor. Frontiers of fitness. Springfield (IL): CC Thomas; 1971. p. 280-94.

Borg GA. Psychophysical bases of perceived exertion. Med Sci Sports Exerc 1982;14:377-381.

Bouhlel E, Jouinia A, Gmada N, Nefzi A, Ben Abdallah K, Tabka Z. Heart rate and blood lactate responses during Taekwondo training and competition. Sci Sports 2006;21:285-90.

Chamari K, Padulo J. 'Aerobic' and 'Anaerobic' terms used in exercise physiology: a critical terminology reflection. Sport Med - Open 2015;1:9.

Chen HI. Effects of 30-h sleep loss on cardiorespiratory functions at rest and in exercise. Med Sci Sports Exerc 1991;23:193-198.

Chtourou H, Hammouda O, Aloui A, Souissi N. Effect of time-of-day on muscle fatigue: a review. J Nov Physiother 2013;3:160.

Davenne D. Sleep of athletes-problems and possible solutions. Biol Rhythm Res 2009;40:45-52.

Goodman J, Radomski M, Hart L, Plyley M, Shephard RJ. Maximal aerobic exercise following prolonged sleep deprivation. Int J Sports Med 1989;10:419-423.

Hammouda O, Chahed H, Chtourou H, Ferchichi S, Miled A, Souissi N. 
Morning-to-evening difference of biomarkers of muscle injury and antioxidant status in young trained soccer players. Biol Rhythm Res 2012;43:431-438.

Horne JA, Ostberg O. A self-assessment questionnaire to determine morningness-eveningness in human circadian rhythms. Int J Chronobiol 1976;4:97-110.

Irwin M, McClintick J, Costlow C, Fortner M, White J, Gillin JC. Partial night sleep deprivation reduces natural killer and cellular immune responses in humans. FASEB J 1996;10:643-653.

Jarraya M, Jarraya S, Chtourou H, Souissi N, Chamari K. The effect of partial sleep deprivation on the reaction time and the attentional capacities of the handball goalkeeper. Biol Rhythm Res 2013;44:503-510.

Juliff LE, Halson SL, Peiffer JJ. Understanding sleep disturbance in athletes prior to important competitions. J Sci Med Sport 2015;18:13-18.

Krustrup P, Mohr M, Amstrup T, Rysgaard T, Johansen J, Steensberg A, Pedersen PK, Bangsbo J. The yo-yo intermittent recovery test: physiological response, reliability, and validity. Med Sci Sports Exerc 2003;35:697-705.

Martin BJ, Chen HI. Sleep loss and the sympathoadrenal response to exercise. Med Sci Sports Exerc 1984;16:56-59.

Martin BJ, Gaddis GM. Exercise after sleep deprivation. Med Sci Sports Exerc 1981;13:220-223.

Mejri MA, Hammouda O, Chaouachi A, Zouaouid K, Ben Rayanad MC, Souissi N. Effects of two types of partial sleep deprivation on hematological responses during intermittent exercise: a pilot study. Sci Sports 2014a;29:266-274.

Mejri MA, Hammouda O, Yousfi N, Haddad M, Souissi N. Sleep, sleep loss and performance in Taekwondo competition. In: Haddad M. editor. Performance optimization in Taekwondo: from laboratory to field. Heathrow (UK): OMICS International; 2014b. p. 1-10.

Mejri MA, Hammouda O, Yousfi N, Zouaoui K, Ben Rayana MC, Chaouachi A. Driss T, Souissi N. One night of partial sleep deprivation affect biomarkers of cardiac damage, but not cardiovascular and lipid profiles, in young athletes. Biol Rhythm Res 2015;46:715-724.

Mejri MA, Hammouda O, Zouaoui K, Chaouachi A, Chamari K, Ben Rayana MC, Souissi N. Effect of two types of partial sleep deprivation on Taekwondo players' performance during intermittent exercise. Biol Rhythm Res 2014c;45:17-26.

Meney I, Waterhouse J, Atkinson G, Reilly T, Davenne D. The effect of one night's sleep deprivation on temperature, mood, and physical performance in subjects with different amounts of habitual physical activity. Chronobiol Int 1998;15:349-363.

Mougin F, Bourdin H, Simon-Rigaud ML, Nguyen NU, Kantelip JP, Davenne D. Hormonal responses to exercise after partial sleep deprivation and after a hypnotic drug-induced sleep. J Sports Sci 2001;19:89-97.

Mougin F, Simon-Rigaud ML, Davenne D, Renaud A, Garnier A, Kantelip JP, Magnin P. Effects of sleep disturbances on subsequent physical performance. Eur J Appl Physiol Occup Physiol 1991;63:77-82.

Oliver SJ, Costa RJ, Laing SJ, Bilzon JL, Walsh NP. One night of sleep deprivation decreases treadmill endurance performance. Eur J Appl Physiol 2009;107:155-161.

Omiya K, Akashi YJ, Yoneyama K, Osada N, Tanabe K, Miyake F. Heartrate response to sympathetic nervous stimulation, exercise, and magnesium concentration in various sleep conditions. Int J Sport Nutr Exerc Metab 2009;19:127-135.

Plyley MJ, Shephard RJ, Davis GM, Goode RC. Sleep deprivation and cardiorespiratory function. Influence of intermittent submaximal exercise. Eur J Appl Physiol Occup Physiol 1987;56:338-344.

Racinais S, Hue O, Blonc S, Le Gallais D. Effect of sleep deprivation on shuttle run score in middle-aged amateur athletes. Influence of initial score. J Sports Med Phys Fitness 2004;44:246-248.

Reilly T, Edwards B. Altered sleep-wake cycles and physical performance in athletes. Physiol Behav 2007;90:274-284

Ricardo JS, Cartner L, Oliver SJ, Laing SJ, Walters R, Bilzon JL, Walsh NP. No effect of a 30-h period of sleep deprivation on leukocyte trafficking, neutrophil degranulation and saliva IgA responses to exercise. Eur J Appl Physiol 2009;105:499-504.

Souissi N, Chtourou H, Aloui A, Hammouda O, Dogui M, Chaouachi A, Chamari K. Effects of time-of-day and partial sleep deprivation on short-term maximal performances of judo competitors. J Strength Cond Res 2013;27:2473-2480.

Souissi N, Souissi M, Souissi H, Chamari K, Tabka Z, Dogui M, Davenne D. Effect of time of day and partial sleep deprivation on short-term, high-power output. Chronobiol Int 2008;25:1062-1076.

Xie L, Kang H, Xu Q, Chen MJ, Liao Y, Thiyagarajan M, O’Donnell J, Christensen DJ, Nicholson C, Iliff JJ, Takano T, Deane R, Nedergaard $\mathrm{M}$. Sleep drives metabolite clearance from the adult brain. Science 2013;342:373-377. 